\section{P90 IMPLEMENTING TRIAGE IN A BUSY HOSPICE TEAM}

Andrew Knight Princess Alice Hospice, Surrey, UK

10.1136/bmjspcare-2011-000105.90

The number of referrals to the Princess Alice Community Team continue to rise and, while it is apparent that the percentage of referrals for patients with non-malignant disease has not increased, the complexity of many referrals and the chronicity of cancer means that patients are referred for longer term care.

The Community Team has been responsive but the need to see a patient as urgently as the referrer perceives has placed undue stress on the Clinical Nurse Specialists (CNS). The referral process was uncontrolled.

We therefore employed a triage nurse to:

- Assess all referrals for appropriateness and urgency

- Gather information about new patients before they are formally assessed

- Respond quickly to urgent referrals

- Speak to patients and carers, introduce the service and gain a sense of their needs

- Assess non-urgent referrals, managing initial new patient needs through liaison with the primary care professionals

- Assign patients to other departments, for example, therapists or day hospice if primary request is for this intervention

- Keep a telephone only contact patient caseload

- Record and audit new referrals and outcome of triage process

The post has been a great success. An additional post has been made.

- CNSs have not felt burdened by new referrals arriving with little information. The triage nurse can obtain copies of clinic letters, imaging reports discharge summaries etc.

- Patients have felt welcomed and their cares listened to, an introduction to the service has been undertaken. They have been reassured.

- Some patients have been 'referred back' to the referrer or GP as not appropriate or, more commonly, have been held on the triage caseload until a time that requires a CNS visit. 
Stats on patterns of referral, outcomes and 'refer backs' will be provided as well as a discussion on future developments. 